\title{
The Relationship of Occupational Stress, Physical Fitness Levels, Body Fat Percentage and Absenteeism
}

\author{
Spanos K*, Strigas A, Travlos A, Kipreos G
}

Department of Sport Organization and Management, Faculty of Human Movement and Quality of Life Sciences, University of Peloponnese, Sparta, Greece

\author{
DOI: $10.36348 /$ jaspe.2020.v03i02.001 \\ | Received: 28.01.2020 | Accepted: 04.02.2020 | Published: 09.02.2020
}

*Corresponding author: Spanos K

\section{Abstract}

Obesity and health problems have been observed that reduces productivity and increases absenteeism. Although the relationship between health and productivity is strong, few studies have examined whether the level of obesity and physical activity affect the absence from work and how the health-related habits are affected. The aim of this study was to examine the relations between job stress body fat, physical fitness levels, health-related habits and absenteeism, among 440 office workers of public sector and private sector companies of Greece employee population. Significant differences observed in the absence days between normal weight $(\mathrm{NW})$ individuals $(3.07 \pm 1.7)$, overweight $(8.56 \pm 3.08)$ and obese $(12.62 \pm 10.1)$. The regression analysis model present an $\mathrm{R}$ value of $0.814, \mathrm{p}<0.05$ and shows that $65.8 \%$ of variance in absenteeism is being explained by body fat percentage, $\mathrm{VO}_{2}$ max flexibility level and the job stressors role and demand In addition, NW employees consume significantly less fast food meals $(2.26 \pm 1.76)$ a week compared to overweight $(3.72 \pm$ $1.91)$ and obese $(3.54 \pm 1.61)$. This study provides evidence that obesity and lack of physical activity affect the absent days from work. Higher body fat is also associated with unhealthy attitudes.

Keywords: Job stress, health behaviors, absenteeism, physical activity, obesity.

Copyright @ 2020: This is an open-access article distributed under the terms of the Creative Commons Attribution license which permits unrestricted use, distribution, and reproduction in any medium for non-commercial use (NonCommercial, or CC-BY-NC) provided the original author and source are credited.

\section{INTRODUCTION}

Lack of regular physical activity is associated with many negative health effects. Studies have shown that overweight and physical inactivity are causes of chronic illnesses / pathologies [1] and risk factors for employee mental health [2]. Therefore, programs to promote and increase physical activity can be important in support of employee health and well-being in the workplace [3]. There is increasing evidence evaluating the effectiveness of physical activity on work-related outcomes, such as job satisfaction, work stress, productivity, work ability, and sickness absence. Increased levels of physical activity have proven positive for physical health and mental well-being. Physical activity is also known to affect work-related outcomes, such as reduced absenteeism. Absence due to illness is a major public health problem with a profound economic impact on society and there may be many that can benefit from physical activity interventions aimed at preventing long-term absenteeism. Examining the relationship between physical activity and absenteeism is therefore important as it can offer benefits to organizations worldwide. Systematic review data show that physical activity is effective in reducing sickness absence [4] However, several studies indicate a number of methodological concerns, such as the lack of a description of the physical activity program in intervention studies and the use of self-reported physical activity in observational studies. We conclude that, overall, the available data provide limited support that physical activity is effective in reducing sickness absence due to the poor quality of many of these studies. It is well known from valid reviews that health promotion programs designed specifically for employees, have a positive impact on the health and wellbeing of workers. Promoting physical activity programs aimed at enhancing the health or fitness of employees has become particularly popular in all areas of work [3,5]. Work stress and poor quality of life can lead to disruption and inefficiency in jobs and premature exhaustion. Recent evidence has shown that specific stress interventions lead to better physical and mental health of employees [6,7]. Specifically, Ohta et al., [7] showed that physical activity increased work ability by reducing absenteeism and improving job ability prediction. In this context, effective exercise and wellness programs to reduce weight, improve physical 
fitness and improve work-related stress and quality of life for workers are absolutely essential.

The literature review also revealed that overweight and obese employees had higher levels of absenteeism and higher sick leave or disability use. The overweight and obese individual also presented more workplace injuries. From an economic point of view, the data analysis showed that health care costs were higher for employees with higher body mass indices [8].

According to a study conducted by Goetzel et al., [9] it was recognized that obese workers had $20 \%$ more medical visits and $26 \%$ more emergency visits than normal-weight employees. Gates et al. [10] found that extremely or moderately obese workers have higher absenteeism rates and showed significantly fewer productivity levels than mildly obese workers. In contrast, in a recent study, the results indicated that obese employees who reduced their weight, they also experienced reduced presenteeism [11]. Although these results should be interpreted with some caution, given the small number of observations per country, the evidence presented suggests that there are interesting geographical patterns in the way obesity affects individuals' self-perceived health, possibly linked to differences in beliefs about the harms of obesity and rates or the value of life.

From the above, it is understood that obesity and lack of physical activity has significant medical and health costs. Although the relationship between health and productivity is robust, few studies have studied whether the level of obesity and activity affect the absence from work due to illness and if these factors interact with health behaviors. The relationship between exercise and work-related absenteeism, efficiency, job satisfaction and employee fatigue has focused primarily on participation rates in the exercise and not on the quantity and quality of the exercise. While previous research indicates that physical activity levels are associated with improved productivity, satisfaction and presence relationships are vulnerable, as only employees' participation in fitness centers and not fitness levels. The purpose of this research was to examine whether obesity, and in particular body fat percentage, as well as the level of aerobic fitness, flexibility and strength affect days of absence from the workplace due to illness. The second goal was to examine if there is a structural model between various stressors, obesity and fitness levels.

\section{MATERIAL AND METHODS}

In this cross-sectional study, a random sample taken out of public sector and private sector companies of Greece employee population. The sample consisted of 440 office workers age (means \pm SD, $42.3 \pm 4.6$ years and BMI $24.5 \pm 4.2$ ), working 40 hours a week. The study focused on 239 women, age $43 \pm 4$ years and
BMI $24.2 \pm 4.2$ and 201 men age $41 \pm 5$ years and BMI $25.4 \pm 4.1$ (means \pm SD). Health \& Safety Executive Management Standards Indicator Tool (HSE-MS) questionnaire was used to collect baseline data and examine levels of job stress. The HSE-MS is a closedended questionnaire consisting of 35 questionnaire items that examine $7 \mathrm{key}$ and important risk factors directly related to work stress: demands, control, manager's support, peer support, relationships, role and change. Demands include issues such as workload, work standards, and the work environment. The control assesses whether the employee is taking the initiative and has a say in the way his work is performed. Support includes the encouragement, privileges and resources provided by the organization, management and colleagues. Relationships include promoting positive work to avoid conflict and tackling unacceptable behavior. The role indicates whether employees understand their role in the organization and whether the organization ensures that they do not have contradictory roles. Change assesses how organizational change (large or small) is managed and communicated to the organization. Scores are scored on a 5-point Likert-type scale (1- never, 2- rarely, 3- sometimes, 4often, 5- always), with a "1" being an inappropriate result and a "5" a desired outcome. The above results provide information on how each individual responds to stressors.

Absenteeism was assessed by a single question, evaluating the frequency of not being at work, due to physical or mental illness, during the preceding year. Illness absence data were registered during 12 months' follow-up. Height and body weight were measured according to a standard protocol by a trained dietician, and an exercise physiologist. Weight and height were measured with clothes and shoes removed, using a SECA scale (seca 899). Body fat percentage was measured by the method of 7 skinfolds (triceps, oblique, abdomen, chest, abdominal, thigh, axillary) using a Harpenden-type dermatometer - and using specific equations [21]. Finding the maximum oxygen consumption (vo2max) was done with a specific protocol. Specifically, after a 5-minute warm-up, the testers started at a low initial speed $(4 \mathrm{~km} \mathrm{/} \mathrm{h}$ for gymnastics, $6 \mathrm{~km} \mathrm{/} \mathrm{h}$ for workouts), and then the intensity was gradually increased every minute in order to exhaust the testers at 8-12 minutes. The highest oxygen value recorded for 1' during the test was also the maximum oxygen uptake. The following criteria were used to terminate the fatigue test: i) VO2max plateau (VO2 rise $<2.5 \mathrm{ml} / \mathrm{kg} / \mathrm{min}$, ii) maximum heart rate +-10 beats / min from 220 and iii) RQ> 1.10 [12].

A sit-and- reach test was used to measure hamstring and low back flexibility. An -1 maximum repetition- bench press and squat test was used to assess maximum muscular strength. Demographic characteristics were also assessed. Other baseline characteristics were Body Mass Index (BMI), weight, 
height, sex and age. The questionnaire included a letter explaining the purpose of the study. In addition, employees were asked to complete the questionnaire without reporting any identifying information.

Average, standard deviations and correlations between variables were examined, while one-way ANOVA were used. Also, Stepwise regression analyses using backward elimination were utilized to determine which health-related fitness variables, job stressors and absenteeism. The probability level was set at $\mathrm{p}<0.05$ to reach statistical significance. For the statistical analysis, the Statistical Package for Social Sciences (SPSS 24.0) was used [13].

\section{FINDINGS AND DISCUSSION}

The purpose of this study was to examine the correlations between body fat percentage, vo2max, health-related habits and absenteeism. Additionally, it has been examined whether the level of obesity and physical activity affect the absence from work and how the health-related habits are affected. Body composition, cardiorespiratory endurance, muscular strength, job stress, and absenteeism were examined. Means and standard deviations of these variables are presented in Table-1.

The results of the present study presents the differences in days of absenteeism, vo2max, fast food and alcohol consumption among normal weight (NW), overweight and obese employees. There was a statistically significant difference between the 3 groups on days of absence and physical activity. In junk food consumption, there was no significant difference between overweight and obese people, but normal weight people consumed significantly less junk food than the other 2 groups (Figure-2).

Table-1: Means and Standard Deviations for Fitness, body composition and job stress variables

\begin{tabular}{|c|c|c|c|}
\hline Variable & $\frac{\text { Total }(n=440)}{\underline{\text { Mean } \pm \text { SD }}}$ & $\frac{\text { Males }(n=201)}{\underline{\text { Mean } \pm \text { SD }}}$ & $\begin{array}{c}\text { Females }(n=239) \\
\underline{\text { Mean } \pm \text { SD }}\end{array}$ \\
\hline Age (years) & $42.3 \pm 4.6$ & $41 \pm 5$ & $43 \pm 4$ \\
\hline BMI & $24.5 \pm 4.2$ & $25.4 \pm 4.1$ & $24.2 \pm 4.2$ \\
\hline$\%$ Fat & $26.8 \pm 6.5$ & $22 \pm 6.2$ & $28.2 \pm 5.8$ \\
\hline $\operatorname{VO} 2 \max (\mathrm{ml} / \mathrm{kg} / \mathrm{min})$ & $31.2 \pm 3.7$ & $32.8 \pm 3.9$ & $30.8 \pm 3.6$ \\
\hline Strength $(\mathrm{kg})$ & $42.9 \pm 14.3$ & $59 \pm 19.9$ & $38.2 \pm 7.1$ \\
\hline Demands & $3.1 \pm 0.7$ & $3.2 \pm 0.7$ & $3.1 \pm 0.7$ \\
\hline Control & $3.5 \pm 0.6$ & $3.5 \pm 0.5$ & $3.6 \pm 0.6$ \\
\hline Manager's support & $3.7 \pm 0.8$ & $3.8 \pm 0.6$ & $3.7 \pm 0.8$ \\
\hline Peer support & $3.9 \pm 0.6$ & $3.9 \pm 0.6$ & $4 \pm 0.6$ \\
\hline Relationships & $4.1 \pm 0.6$ & $4.1 \pm 0.6$ & $4.1 \pm 0.6$ \\
\hline Role & $4.3 \pm 0.5$ & $4.3 \pm 0.4$ & $4.3 \pm 0.6$ \\
\hline Change & $3.2 \pm 0.8$ & $3.2 \pm 0.8$ & $3.2 \pm 0.7$ \\
\hline Absenteeism (days) & $4.8 \pm 3.1$ & $4 \pm 3$ & $5.2 \pm 3$ \\
\hline
\end{tabular}

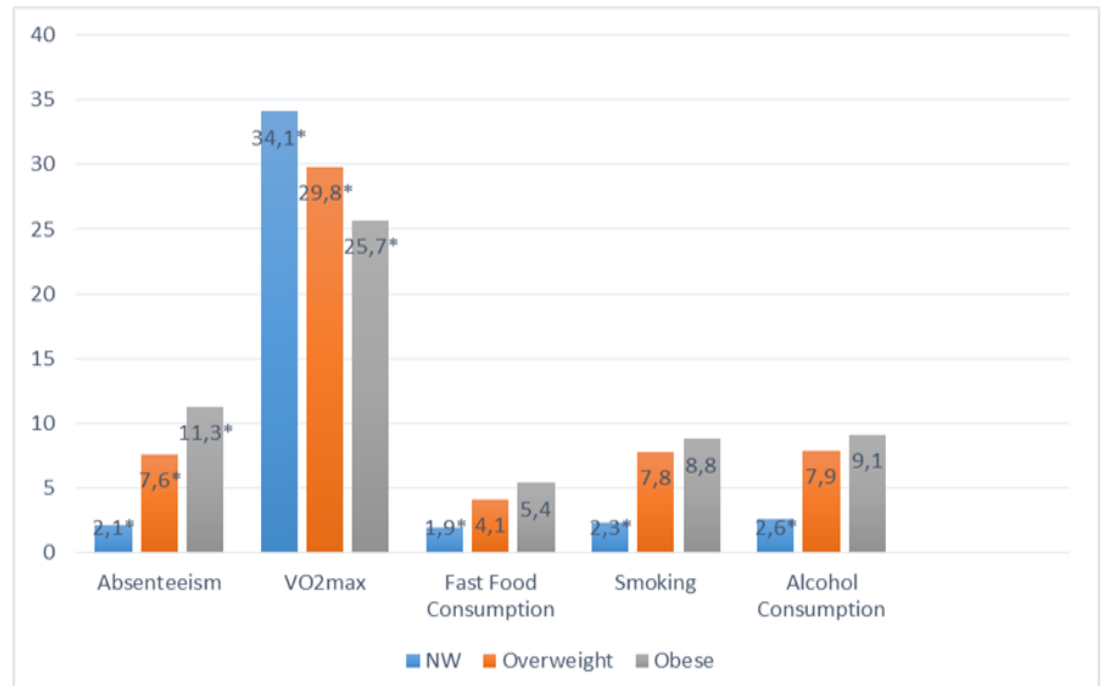

Fig-2: Days of absenteeism, physical activity, fast food and alcohol consumption, smoking for different groups 
There was a significant difference in the absence days between NW individuals (2.1 days), overweight (7.6 days) and obese (11.3 days). The data on health-related habits showed that people with higher body fat percentage adopted more unhealthy habits. Specifically, NW employees showed significantly vo2max values $(34.1 \mathrm{ml} / \mathrm{kg} / \mathrm{min})$ than overweight $(29.8$ $\mathrm{ml} / \mathrm{kg} / \mathrm{min})$ and obese $(25.7 \mathrm{ml} / \mathrm{kg} / \mathrm{min})$. Additionally, NW employees, consume significantly less fast food and alcohol per week, compared to overweight and obese employees. In addition, NW employees smoke less cigarettes per day overweight and obese subjects.

Correlations ranged from -0.670 to 0.745 indicating that these variables measured relatively independent constructs. In order to further examine the multivariate relationship, stepwise regression analyses was performed. Separate stepwise regressions using backward elimination were performed on each of the job variable measures (i.e., job stress and absenteeism) and the four fitness measures. Employees' body fat percentage remained in the equation with a beta weight of $0.264, p<0.001$. Employees' cardiovascular endurance $\left(\mathrm{VO}_{2} \mathrm{max}\right)$ remained in the equation with a beta weight of $0.301, p<0.001$. Employees' flexibility (sit-n-reach) remained in the equation with a beta weight of $0.15, \mathrm{p}<0.001$. Employees' job stressor «role» remained in the equation with a beta weight of 0.573 , $\mathrm{p}<0.001$ and «demand» with a beta weight of 0.413 , $\mathrm{p}<0.01$. The regression analysis model present an $\mathrm{R}$ value of $0.814, \mathrm{p}<0.05$ and shows that $65.8 \%$ of variance in absenteeism is being explained by body fat percentage, $\mathrm{VO}_{2} \max$ flexibility level and the job stressors role and demand.

The present study shows that body fat percentage correlates with days of absence from work due to physical or mental illness. A correlation also occurs between physical activity and absence days. As expected, physical activity affects the body fat percentage, with the most trained individuals having a lower body fat percentage. It also appears that eating junk food and sleeping hours, as isolated factors, do not affect days of absence. A finding of the research is that obese people also adopt more unhealthy behaviours. In particular, obese and overweight people exercise less, consume more junk food and alcohol and smoke more. They also suffer from musculoskeletal problems and health problems in general. Although overweight and obese individuals presented more musculoskeletal pain, the rate is also quite high in $\mathrm{NW}$ employees too, as about one in two people suffers from musculoskeletal pains. This is probably due to the type of work and other ergonomic factors.

The results of the present research agree with other studies showing that obese employees and workers with higher self-reported BMI values, experienced higher absenteeism rates than overweight or normal weight employees [14]. Gates et al., [10] found that extremely or moderately obese workers have higher absenteeism rates and showed significantly fewer productivity levels than mildly obese workers. The above results are consistent with the current survey, where obese and overweight people were absent more days than normal people from their work. In contrast, the results of the present study do not agree with Gifford's study [11], where the results indicate that obese employees who reduced their weight, they also experienced reduced presenteeism.

Regarding participation in training programs, the results of the present research are in agreement with a study taken place in the Netherlands. Jans et al., [15], found that obese employees were absent 14 days a year more than normal-weight employees. Also, the frequency of absenteeism of more than 7 days was significantly higher. The differences in absenteeism between obese and normal-weight employees were larger for employees who did not exercise regularly. Obese staff is more often and for a long time absent, particularly the people who don't exercise regularly.

Employees' maximum muscular strength, as assessed through a bench press and squat test, didn't influenced days of absenteeism. Employees' cardiovascular endurance assessed through the $\mathrm{VO}_{2}$ max protocol test on a physical work capacity treadmill ergometer test. Employees with higher levels of cardiovascular endurance have greater cardiovascular efficiency in another study involving employees of big technology corporation. Employees indicated decreased anxiety and increased job satisfaction upon completion of a 14-week fitness program [16]. This finding illustrates the influence of aerobic exercise to benefit employees' sense of wellness status and reduce absenteeism. Possible explanations for these findings could embody a reduction in physical fatigue thanks to a rise of labor capability, a reduction of minor health problem, and relief from tedium, anxiety or aggression [17]. Employees' flexibility measured through the sitand- reach test that assesses hamstring and low back flexibility. Lack of flexibility in this area may be associated with an increased risk factor for the development of chronic lower back pain [18, 19]. It would seem possible then that employees with less flexibility may have been absent more often due to lower back pain. Although past research has not identified flexibility to correlate with employees' absenteeism, research has indicated relationships between participants in employee fitness programs and lower absenteeism [19]. Wattles et al., showed a correlation between flexibility and absenteeism [19].

Increased fitness levels can cause improved health, and healthier working staff is less likely to be absent. However, this assumption only relates to absences due to medical reasons, yet employees stay away from work for more reasons than simply health problems [19]. Several studies do not indicate positive 
relationships between exercise and absenteeism [17, 18]. Shephard et al. investigated the results of an employee fitness program on absence and productivity. After the completion of a six-month exercise program, exercising working staff developed substantial gains in fitness but no significant difference was observed in absenteeism, between the exercising and the nonexercising groups [17].

An important factor that might influence the results and has been examined by the present investigation is the psychological work stress and job strain effects on body fat and weight loss or gain. In a survey conducted in England, it seemed that job strain (combination of high job demands and low control) created bidirectional effects on obesity. On one hand, job stress seems to drive in weight gain among obese and overweight men employees, while on the other hand, it seems that lean employees lose weight [20]. The present study suggest that job stressors like demands and role, have a significant impact on absenteeism.

\section{CONCLUSION}

Although absenteeism is a multifactorial phenomenon, this study provides evidence that obesity, the lack of physical activity and job stressors demands and role, affect the absent days from work. Higher body fat percentage is also associated with unhealthy attitudes and health problems. In particular, the results of this research showed that there is a significant correlation between high body mass index and days of absence from the workplace. Also, overweight and obese individuals have experienced more musculoskeletal pains and more health problems associated with high mortality rates. Finally, an important finding is that people with high body fat exhibit more unhealthy attitudes, which affects public health. Overweight and obese people tend to be less exercised, smoke more, eat more junk food, and consume more alcohol. This shows that obesity has direct and indirect costs not only for employers but also for health in general.

Planning interventions to tackle this complex health issue, requires a coordinated intervention in the workplace, as people spend most of their time there. Employers also have an economic interest in promoting the health and well-being of their employees. Employers should be encouraged to adopt management programs for obesity, change health-related behaviors and stress regulation. The implementation of wellness programs at workplaces should not only be limited to the provision of exercise programs but should aim at adopting behaviors that contribute to improving the quality of life and increase their potential economic growth.

\section{ACKNOWLEDGMENTS}

This research is co-financed by Greece and the European Union (European Social Fund- ESF) through the Operational Programme «Human Resources Development, Education and Lifelong Learning» in the context of the project "Strengthening Human Resources Research Potential via Doctorate Research" (MIS5000432), implemented by the State Scholarships Foundation (IKY).

\section{REFERENCES}

1. Healy, G. N., Winkler, E. A., Brakenridge, C. L., Reeves, M. M., \& Eakin, E. G. (2015). Accelerometer-derived sedentary and physical activity time in overweight/obese adults with type 2 diabetes: cross-sectional associations with cardiometabolic biomarkers. PloS one, 10(3): $\mathrm{e} 0119140$.

2. Atlantis, E., Chow, C. M., Kirby, A., \& Fiatarone Singh, M. A. (2006). Worksite intervention effects on physical health: a randomized controlled trial. Health Promotion International, 21(3), 191200.

3. Quintiliani, L., Sattelmair, J., \& Sorensen, G. (2007). The workplace as a setting for interventions to improve diet and promote physical activity. Paper presented at: World Health Organization (WHO)/World Economic Forum (WEF) joint event on preventing noncommunicable diseases in the workplace, Dalian, China.

4. Amlani, N. M., \& Munir, F. (2014). Does physical activity have an impact on sickness absence? A review. Sports Medicine, 44(7), 887-907.

5. Moreira-Silva, I., Santos, R., Abreu, S., \& Mota, J. (2014). The effect of a physical activity program on decreasing physical disability indicated by musculoskeletal pain and related symptoms among workers: a pilot study. International Journal of Occupational Safety and Ergonomics, 20(1), 55-64.

6. Tetrick, L. E., \& Winslow, C. J. (2015). Workplace stress management interventions and health promotion. Annu. Rev. Organ. Psychol. Organ. Behav., 2(1), 583-603.

7. Ohta, M., Eguchi, Y., Inoue, T., Honda, T., Morita, Y., Konno, Y., ... \& Kumashiro, M. (2015). Effects of bench step exercise intervention on work ability in terms of cardiovascular risk factors and oxidative stress: a randomized controlled study. International Journal of Occupational Safety and Ergonomics, 21(2), 141-149.

8. Schmier, J. K., Jones, M. L., \& Halpern, M. T. (2006). Cost of obesity in the workplace. Scandinavian journal of work, environment \& health, 5-11.

9. Goetzel, R. Z., Gibson, T. B., Short, M. E., Chu, B. C., Waddell, J., Bowen, J., ... \& DeJoy, D. M. (2010). A multi-worksite analysis of the relationships among body mass index, medical utilization, and worker productivity. Journal of 
occupational and environmental medicine, 52(Suppl 1), S52-58.

10. Gates, D. M., Succop, P., Brehm, B. J., Gillespie, G. L., \& Sommers, B. D. (2008). Obesity and presenteeism: the impact of body mass index on workplace productivity. Journal of Occupational and Environmental Medicine, 50(1), 39-45.

11. Gifford, B. (2015). Unhealthy body weight, illness absence, presenteeism, medical payments, and disability leave: A longitudinal view. Population health management, 18(4), 272-282.

12. ACSM's Guidelines for Exercise Testing and Prescription 10th Edition (2017). Lippincott Williams and Wilkins. Philadelphia, United States.

13. IBM Corp. Released 2016. IBM SPSS Statistics for Windows, Version 24.0. Armonk, NY: IBM Corp

14. Quesenberry, C. P., Caan, B., \& Jacobson, A. (1998). Obesity, health services use, and health care costs among members of a health maintenance organization. Archives of internal medicine, 158(5), 466-472.

15. Jans, M. P., van den Heuvel, S. G., Hildebrandt, V. H., \& Bongers, P. M. (2007). Overweight and obesity as predictors of absenteeism in the working population of the Netherlands. Journal of occupational and environmental medicine, 49(9), 975-980.

16. Pauly, J. T., Palmer, J. A., Wright, C. C., \& Pfeiffer, G. J. (1982). The effect of a 14-week employee fitness program on selected physiological and psychological parameters. Journal of occupational medicine.: official publication of the Industrial Medical Association, 24(6), 457-463.

17. Shephard, R. J., Cox, M., \& Corey, P. (1981). Fitness program participation: its effect on worker performance. Journal of occupational medicine.: official publication of the Industrial Medical Association, 23(5), 359-363.

18. Falkenberg, L. E. (1987). Employee fitness programs: Their impact on the employee and the organization. Academy of Management Review, 12(3), 511-522.

19. Wattles, M. G., \& Harris, C. (2003). The Relationship Between Fitness Levels And Employee's Perceived Productivity, Job Satisfaction, and Absenteeism. Journal of Exercise Physiology Online, 6(1):24-32.

20. Kivimäki, M., Head, J., Ferrie, J. E., Shipley, M. J., Brunner, E., Vahtera, J., \& Marmot, M. G. (2006). Work stress, weight gain and weight loss: evidence for bidirectional effects of job strain on body mass index in the Whitehall II study. International journal of obesity, 30(6), 982-987.

21. Jackson, A. S., Pollock, M. L., \& Ward, A. N. N. (1980). Generalized equations for predicting body density of women. Medicine and science in sports and exercise, 12(3), 175-181. 